\title{
Morphological variations of human adult spleens: A cadaveric study
}

\author{
Kiritharan $\mathrm{A}^{1}$, Samaranayake $\mathrm{UMJE}^{1}$, Anthony $\mathrm{DJ}^{1}$ \\ ${ }^{I}$ Department of Anatomy, Faculty of Medicine, University of Colombo. \\ *abarana2001@gmail.com
}

Spleen is an organ enriched in vascular and lymphoid tissue located in left hypochondrium. It has morphological variations which may be misinterpreted as a disease condition involving the spleen. Therefore, knowledge on such morphological differences in a population is vital to distinguish normal variations from the disease conditions. The objective of this study was to identify the morphological variations such as notches, fissures and lobulations in cadavers. Ten per cent formalin fixed, self-donated cadaveric human spleens $(\mathrm{n}=13)$ in the Department of Anatomy, Faculty of Medicine, University of Colombo were included in the present study. In the study population, male to female ratio was 10: 3 with the age ranging from 26 to 95 years. The morphological features such as shape, notching of borders, fissures and lobulations were photographed, dissected and analyzed according to Michels NA classification. The morphological types of spleens were categorized by measuring hilar lengths. Notches were present in superior $(\mathrm{n}=8)$, inferior $(\mathrm{n}=1)$ or intermediate $(\mathrm{n}=2)$ borders in ten spleens, while notches were absent in three. Five spleens had supernumerary notches. Six spleens contained fissures in both visceral and diaphragmatic surfaces. Four spleens had more than three lobules, while three had more than one hilum. The splenic types included compact, intermediate and distributed, which accounted for four, three and six respectively. Inconstant location of notches, presence of supernumerary notches, fissures and lobules were a common morphological variation observed. 